\title{
Incidence of the Metabolic Syndrome among Patients with Epilepsy Attending a Neuropsychiatric Hospital in Kigali, Rwanda
}

\author{
François Xavier Ndayambaje ${ }^{1}$, Jean Bosco Gahutu ${ }^{2}$, Simon Peter Rugera ${ }^{3}$, Bernard Natukunda ${ }^{4}$ \\ ${ }^{1,3,4}$ Department of Medical Laboratory Science, Mbarara University of Science and Technology (MUST), P.O.Box 1410, \\ Mbarara, Uganda \\ ${ }^{2}$ Department of Physiology, Faculty of Medicine, University of Rwanda (UR), P.O. Box 3286, Kigali, Rwanda
}

\begin{abstract}
Background \& Aims: Metabolic syndrome (MetS), a combination of diverse metabolic disorders (hypertension, diabetes mellitus, high triglycerides, increased waist circumference, and low high density cholesterol, HDLc), is a well known public health problem worldwide, and its prevalence is increasing dramatically. MetS is a confirmed great risk factor for cardiovascular diseases.

Presently, limited information exists about incidence and the risk factors associated with metabolic syndrome (MetS) in patients with epilepsy. We prospectively estimated the incidence of MetS in patients with epilepsy.

Methods: We recruited 322 participants, 161 patients with epilepsy and 161 healthy volunteers all of them free of any MetS criteria at the baseline and followed-up them for one year. New onset cases of MetS were defined according to the updated National Cholesterol Education Program (NCEP) Adult Treatment Panel III (ATPIII) criteria.

Results: Over a 1-year follow-up, we observed 8 incident cases of MetS (7 individuals in patients group and 1 individual in control group) resulting in an annual cumulative incidence rate of $2.5 \%$. We observed 9 cases losses to follow up. Patients with epilepsy presented a higher risk of developing MetS $(\mathrm{RR}=7.00,95 \% \mathrm{CI}: 0.891-1.67, \mathrm{p}=0.032)$ than in control group. Sedentariness was associated with higher risk for MetS (hazard ratio, HR=6.537, 95\%; confidence interval, C.I=1.269-33.685, $p$ value $=0.025$.
\end{abstract}

Conclusion: Anti-epileptic therapy combined with sedentariness increases the risk of developing MetS among patients with epilepsy. Holistic clinical management of patients with epilepsy will significantly contribute to MetS prevention.

KEYWORDS: Metabolic syndrome, epilepsy, incidence, anti-epileptic drugs

\section{INTRODUCTION}

Metabolic syndrome (MetS) which is a cluster of obesity, high blood pressure, dyslipidemia and impairment of insulin metabolism,[1] is becoming a serious global health threat.[2] MetS is a well known great risk factor for developing cardiovascular diseases[3][4]-[7] and cerebrovascular diseases. [8] Due to westernization of life style, its prevalence and incidence are increasing dramatically.[9] Much interest has been put in studying the incidence of MetS in general population[10] however fewer works have focused on investigating the incidence in specific populations who have other underlying conditions.[11] One of many other frequent medical conditions is epilepsy which is one of the most prevalent neurological conditions that knows no age, racial, social class, geographic, or national boundaries.[12] Some researchers have shown that lifestyle devoid of exercises and use antiepileptic drugs (AEDs) contribute significantly in the occurrence of MetS in patients with epilepsy.[13]. AEDS which are commonly used in Rwanda are: valproic acid (depakene), lorazepam (ativan), carbamazepine (tegretol), leveracetam (spritam), diazepam (valium), phenytoin (dilintin), phenobarbital (phenobarbitone or luminal), clonazepam (klonopin) and topomax (topiramate).

Several diagnostic criteria have been proposed for the clinical diagnosis of the MetS, but the most commonly used criteria for definition at present are from the World Health Organization (WHO) and National Cholesterol Education Program (NCEP) Adult Treatment Panel III (ATPIII)[14]. In this study we used the latter because we were dealing with adult people.

This study aimed to estimate the incidence of MetS in patients with epilepsy receiving regular care. Information regarding the incidence of MetS in such group of population is useful to get a better comprehension of course of MetS and cardiovascular risk in people with epilepsy. 


\section{International Journal of Current Science Research and Review}

ISSN: 2581-8341

Volume 04 Issue 05 May 2021

DOI: 10.47191/ijcsrr/V4-i5-03, Impact Factor: 5.825

IJCSRR@ 2021

Www.ijcsrr.org

\section{MATERIAL AND METHODS}

\section{Study participants}

This was a prospective cohort study whose aim was to estimate the incidence of MetS in Rwandan patients with epilepsy. The study was carried out in the Out-Patients' Department (OPD) of Ndera Neuro-Psychiatric Hospital, in Kigali, Rwanda. The study duration was 12 months from December 2018 to December 2019 during which we calculated the incidence of MetS. According to sample size calculation, the target sample size was 322 study participants.

The recruitment process was based on convenience sampling method. We approached and invited potential research candidates. However, certain categories of the people were not invited to participate in our study like people with malignancies, pregnancy, mental or legal incapacities that prevented them from giving a valid consent or hindered them from full participation in the study process or strict adherence to study protocol. For those who were invited to participate, we first inquired from them if they already had component of MetS like high blood pressure or diabetes mellitus by giving them a self-questionnaire to fill in relevant information. If an individual could self report as having any component of MetS or was found to have it after medical and laboratory examination he was excluded from our study. We carefully selected and screened our study participants in that manner until we reached the targeted simple size. Therefore in total 322 participants signed the inform consent and follow-up forms; 161 of them were patients with epilepsy receiving monotherapy anti-epileptic drugs and the remaining 161 participants were healthy volunteers all of them free of any MetS criteria at the baseline

Data on lifestyle and metabolic risk factors were collected for both groups and participants were requested to come after six months meaning twice per the period of follow-p. As we used NCEP- ATPIII criteria to confirm MetS, a study participant was diagnosed with epilepsy if he developed three or more of the following risk factors which were absent at baseline: (i)Waist circumference $>102 \mathrm{~cm}$ (40.2 in) in men and $>88 \mathrm{~cm}$ (35.6 in) in women, (ii) Serum triglycerides $\geq 150 \mathrm{mg} / \mathrm{dl}(1,7 \mathrm{mmol} / \mathrm{L})$, (iii) Blood pressure Systolic BP $\geq 130$ and/or diastolic BP $\geq 85 \mathrm{mmHg}$, (iv) HDL cholesterol $<40 \mathrm{mg} / \mathrm{dl}(<1.03 \mathrm{mmol} / \mathrm{L}$ ), in men and $<50 \mathrm{mg} / \mathrm{dl}(1.29 \mathrm{mmol} / \mathrm{L})$ in women, and (v) Fasting plasma glucose $\geq 110 \mathrm{mg} / \mathrm{dl}) \quad(>6.1 \mathrm{mmol} / \mathrm{L})$.

\section{Data collection}

Before the commencement of the study, patients and healthy volunteers who voluntarily agreed to participate in the study signed a written consent form following a detailed explanation on the study. Participants were then interviewed and examined in a special room for privacy and confidentiality. Demographic details (names, sex, age and marital status), risk factors (diabetes, hypertension, physical activity and smoking) and anthropometric measurements (height, weight and waist circumference) were collected using data collection forms. The interview was carried out by the Principal Investigator (PI) and one Research Assistant (RA) who had been trained before commencing the study.

\section{Anthropometric measurements}

Waist circumference was taken without outer clothing, using a non-stretchable tape measure at the level of the uppermost edge of the hip bone on abdomen with the tape parallel to the ground and recorded to the nearest 0.5 centimeter. Blood pressure (BP) was taken from the arm (brachial artery) for all respondents on the first encounter by using a digital sphygmomanometer. BP measurements were performed in the sitting position with the arm supported at heart level and repeated after 5 minutes; the average of the two measurements was taken as the correct BP for the individual. To calculate the body mass index (BMI), subjects were weighed (in kilograms) on a weighing scale while fully dressed but without shoes. Height (in meters) was measured without shoes by using a stadiometer. The BMI was calculated as the weight divided by the height squared $\left(\mathrm{kg} / \mathrm{m}^{2}\right)$.

\section{Biochemical tests}

Blood samples were collected with BD Vacutainer ${ }^{\circledR}$ venous blood collection tubes. Five milliliters of venous blood were taken from the antecubital fossa and placed in empty sterile vacutainer tubes. The blood samples were separated by centrifugation at $1000 \times \mathrm{g}$ for $15 \mathrm{~min}$ and the supernatant serum was collected. Serum levels of lipids Triglycerides (TG), Total Cholesterol (TC), High Density Lipoprotein Cholesterol (HDL-C)], Blood glucose were measured using commercially available kits (Roche System Reagents) by a Cobas C 311 autoanalyzer using appropriate quality control measures for the reagents and autoanalyser. 


\section{International Journal of Current Science Research and Review}

ISSN: 2581-8341

Volume 04 Issue 05 May 2021

DOI: 10.47191/ijesrr/V4-i5-03, Impact Factor: 5.825

IJCSRR@ 2021

WWw.ijcsrr.org

\section{Statistical analysis}

Data were entered and analyzed using the Statistical Package for Social Science (SPSS) version 24.0 software (SPSS Inc. Chicago, IL). Cumulative incidence of MetS was expressed as the number of cases of MetS per 1,000 patient years of follow-up. On another hand, the annual incidence rate was expressed per 1,000 per person-years of MetS among patients with epilepsy. We calculated odds ratio (OR), relative risk (RR) and hazard ratios (HR) for developing MetS in initially MetS free patients and controls. Furthermore, survival analysis was performed to examine the interval between entry onto study and occurrence of MetS. Kaplan-Meier method and the log-rank test (Mantel-Cox) were used to compare event-free survival among patients with epilepsy and controls. We used proportional hazards analysis to generate hazard ratios (HRs) and 95\% CI for the unadjusted association between baseline characteristics and the occurrence of MetS. HRs were then adjusted for the confounding effects of other baseline characteristics, including familial history of diabetes or high blood pressure, age, sex, smoking status and level of regular physical activities (Cox regression analysis). For tests and models, a $p$-value of less than 0.05 was taken for statistical significance. All tests for statistical significance were two-tailed, confidence intervals (CIs) were set at $95 \%$.

\section{Ethical clearance}

Before the commencement of the study, ethical clearance was sought from Mbarara University of Science and Technology - Research Ethics Committee (MUST - REC) in Mbarara, Uganda. Ethical approval was then secured from both Rwanda National Health Research Committee (NHRC), Rwanda National Ethics Committee (RNEC) and Ndera NeuroPsychiatric Hospital Ethic Committee. We conducted this study in accordance with the guidelines of the Declaration of Helsinki on Ethical Principles for Medical Research Involving Human Subjects. Information concerning the study was given to all study participants prior to the interviews and sample collection. A written and duly signed consent was obtained from all participants. Confidentiality was ensured for all individuals who participated in the study by removing of all identifiers at the time of data entry. The participation was fully voluntary devoid of any kind of coercion and no participant was paid for their involvement in the study

\section{RESULTS}

After a 1-year follow-up period (12 months), eight individuals [7 (2.2\%) individuals in cases group and $1(0.3 \%)$ individual in control group] developed MetS. Therefore, the cumulative incidence of MetS was $2.4 \%(8 / 322)$ with a total of 7 cases (2.1\%) occurring among the patients group and with one case $(0.3 \%)$ occurring in healthy volunteer group with a relative risk (RR) of 8 (2.4/0.3). Nine (2.8\%) subjects were lost to follow-up i.e. cases, $n=5(1.8 \%)$ and controls, $n=4(1.2 \%)$.

Adjusting for different length of follow up until occurrence of the event/censoring, person-months methods allowed us to estimate that the rate of incidence of MetS was 4 MetS cases per 1000 per person-month in patient group; and 0.5 MetS case per 1000 per person-month in healthy volunteer group.

The cumulative incidence of single component of MetS is shown in table 4. Among the 8 new cases of metabolic syndrome, 5 $(1.5 \%)$ subjects had high blood pressure, 6 (1.9.5\%) had hypertriglycerides, 4 (1.2\%) had low HDL, 6 (1.9.5\%) had high blood glucose and $3(0.9 \%)$ had high waist circumference

In the multivariate model, the relative risk (RR) of developing MetS was higher in patient group ( $\mathrm{RR}=7.00,95 \% \mathrm{CI}$ : 0.891 - 1.67, $\chi^{2}=4.615$, $1 \mathrm{df}, p=0.032$ ) than in control group. Kaplan-Meier survival curves of the two groups were significantly different with log-rank (Mantel-Cox) test $(\mathrm{p}=0.028)$. After verifying the effect of each variable (family history of diabetes mellitus, family history of hypertension, male sex, old age, sedentary lifestyle and smoking habits) individually on the occurrence of MetS, a multiple Cox regression analysis was performed. The outcome of this showed that the only predictor of occurrence of MetS was sedentary lifestyle, (hazard ratio, HR=6.537, 95\%; confidence interval, C.I=1.269-33.685, $p$ value $=0.025$ ). Interactions of others predictors failed to reach the significance; $p$ value $>0.050$. 


\section{International Journal of Current Science Research and Review}

ISSN: 2581-8341

Volume 04 Issue 05 May 2021

DOI: 10.47191/ijesrr/V4-i5-03, Impact Factor: 5.825

IJCSRR@ 2021

www.ijesrr.org

Table 1. Demographic and clinical characteristics of among patients with epilepsy and healthy volunteers at the baselin

\begin{tabular}{|c|c|c|}
\hline Component & $\begin{array}{l}\text { Cases group } \\
(\mathrm{N}=161)\end{array}$ & $\begin{array}{l}\text { Healthy } \\
(\mathrm{N}=161)\end{array}$ \\
\hline Mean $( \pm$ SD $)$, age, years & 39.3 & 40.8 \\
\hline Female n $(\%)$ & $87(54.0 \%)$ & $81(50.3 \%)$ \\
\hline Male, n (\%) & $74(46.0 \%)$ & $80(49.7 \%)$ \\
\hline High abdominal obesity & $0(0 \%)$ & $0(0 \%)$ \\
\hline Mean $( \pm$ SD), blood glucose, $\mathrm{mmol} / \mathrm{L}$ & $5.12 \pm 0.75$ & $5.14 \pm 0.64$ \\
\hline Mean $( \pm \mathrm{SD})$, Low HDL-C, mmol/L & $3.85 \pm 0.98$ & $3.76 \pm 0.82$ \\
\hline Mean $( \pm \mathrm{SD})$, Low HDL-C, mmol/L & $1,20 \pm 0.20$ & $1.23 \pm 0.19$ \\
\hline Mean $( \pm$ SD), High TG, mmol/L & $1.30 \pm 0.62$ & $1.25 \pm 0.53$ \\
\hline Mean $( \pm$ SD $)$, body mass index & $22.6 \pm 1.3$ & $22.74 \pm 1.8$ \\
\hline Mean $( \pm$ SD), Systolic blood pressure, $\mathrm{mm} \mathrm{Hg}$ & $118.4 \pm 15.6$ & $1194 \pm 13.5$ \\
\hline Mean $( \pm \mathrm{SD})$, diastolic blood pressure, $\mathrm{mm} \mathrm{Hg}$ & $814 \pm 11.9$ & $794 \pm 10.8$ \\
\hline
\end{tabular}

Table 2. Demographic and clinical characteristics of among patients with epilepsy and healthy volunteers after one year follow-up

\begin{tabular}{|c|c|c|}
\hline Component & $\begin{array}{l}\text { Cases group } \\
(\mathrm{N}=156)\end{array}$ & $\begin{array}{l}\text { Healthy } \\
(\mathrm{N}=157)\end{array}$ \\
\hline Mean $( \pm \mathrm{SD})$, age, years & 40.1 & 41.6 \\
\hline Male, n (\%) & $91(58.3 \%)$ & $74(47.1 \%)$ \\
\hline Female n $(\%)$ & $65(41.1 \%)$ & $83(53.2)$ \\
\hline High abdominal obesity & $11(3.4 \%)$ & $4(1.2 \%)$ \\
\hline Mean $( \pm \mathrm{SD})$, blood glucose, $\mathrm{mmol} / \mathrm{L}$ & $6.02 \pm 0.75$ & $5.62 \pm 0.64$ \\
\hline Mean $( \pm$ SD), Low HDL-C, mmol/L & $3.85 \pm 0.98$ & $3.76 \pm 0.82$ \\
\hline Mean $( \pm$ SD), Low HDL-C, mmol/L & $1,09 \pm 0.20$ & $1.18 \pm 0.19$ \\
\hline Mean $( \pm$ SD $)$, High TG, mmol/L & $1.54 \pm 0.62$ & $1.25 \pm 0.53$ \\
\hline Mean ( \pm SD), body mass index & $25.6 \pm 1.3$ & $22.74 \pm 1.8$ \\
\hline Mean ( \pm SD), Systolic blood pressure, $\mathrm{mm} \mathrm{Hg}$ & $1254 \pm 15.6$ & $1194 \pm 13.5$ \\
\hline Mean $( \pm \mathrm{SD})$, diastolic blood pressure, $\mathrm{mm} \mathrm{Hg}$ & $894 \pm 11.9$ & $784 \pm 10.8$ \\
\hline
\end{tabular}

Table 3. Incidence of metabolic syndrome among patients with epilepsy and control group after a one year of follow up

\begin{tabular}{llll}
\hline & \multicolumn{4}{l}{ Metabolic syndrome } & Total \\
\cline { 2 - 4 } & Yes & No & $161(50 \%)$ \\
\hline Case group & $7(4.3 \%)$ & $154(95.7 \%)$ & $161(50 \%)$ \\
\hline Control group & $1(0.6 \%)$ & $160(99.4 \%)$ & $322(100 \%)$ \\
\hline Total & $8(2.5 \%)$ & $314(97.5 \%)$ & 3 \\
\hline
\end{tabular}

Table 4. Cumulative incidence of metabolic risk factors among patients with epilepsy and healthy volunteers during one year follow-up

\begin{tabular}{llll}
\hline Component & $\begin{array}{l}\text { Total population } \\
(\mathbf{N = 3 2 2})\end{array}$ & $\begin{array}{l}\text { Cases group } \\
(\mathbf{N = 1 6 1 )}\end{array}$ & $\begin{array}{l}\text { Control group } \\
(\mathbf{N = 1 6 1 )}\end{array}$ \\
\hline High glucose, $\mathrm{n}(\%)$ & $6(1.8 \%)$ & $5(3.1 \%)$ & $1(0.62)$ \\
\hline High abdominal obesity, $\mathrm{n}(\%)$ & $15(0.9)$ & $11(3.4 \%)$ & $4(1.2 \%)$ \\
\hline Low HDL-C, $\mathrm{n}(\%)$ & $4(1.2)$ & $3(1.8)$ & $0(0 \%)$
\end{tabular}




\section{International Journal of Current Science Research and Review}

ISSN: 2581-8341

Volume 04 Issue 05 May 2021

DOI: 10.47191/ijcsrr/V4-i5-03, Impact Factor: 5.825

IJCSRR@ 2021

WWw.ijcsrr.org

\begin{tabular}{llll}
\hline High TG, n (\%) & $6(1.8 \%)$ & $6(3.7 \%)$ & $0(0 \%)$ \\
\hline High BP, n (\%) & $5(1.5 \%)$ & $4(2.4 \%)$ & $1(0.62)$ \\
\hline
\end{tabular}

Components by National Cholesterol Education Programme definition: visceral obesity: waist $>102 \mathrm{~cm}$ in men and $>88 \mathrm{~cm}$ in women; high BP: BP >130 or > $85 \mathrm{mmHg}$ or treatment for hypertension; low HDL-C: HDL-C ,1.036 mmol/121 (40 mg/dl) in men and ,1.7 mmol/121 (50 mg/dl) in women; high TG: serum triglycerides >1.695 mmol/121 (150 mg/dl); high glucose: plasma glucose $>6.1 \mathrm{mmol} / 121(100 \mathrm{mg} / \mathrm{dl})$. BP, blood pressure; HDL-C, high-density lipoprotein-cholesterol; TG, triglyceride

Table 5. Cox regression analysis for incidence of metabolic syndrome in a multivariate model

\begin{tabular}{llll}
\hline Risk factors & HR & $\mathbf{9 5 \%}$ CI & $\boldsymbol{p}$ =value \\
\hline Family history of diabetes mellitus & 0.276 & $0.032-2.355$ & 0.239 \\
\hline Family history of hypertension & 1.646 & $0.321-8.433$ & 0.550 \\
\hline Male sex & 0.274 & $0.054-1.405$ & 0.121 \\
\hline Old age $(\geq 45$ years $)$ & 0.358 & $0.069-1.858$ & 0.222 \\
\hline Sedentary lifestyle & 6.537 & $1.269-33.685$ & $\mathbf{0 . 0 2 5}$ \\
\hline Smoking habits & 0.475 & $0.053-4.290$ & 0.508
\end{tabular}

HR: Hazard ratio, C.I: Confidence interval

\section{DISCUSSION}

Although there is scarcity of literature about the incidence of MetS among people with epilepsy for a comparative assessment, our findings indicate that people with epilepsy are more prone to develop MetS and its components than the general population and that preventive actions should be urgently taken.

Previous studies have reported an association between epilepsy and anti-epileptic drugs with MetS, however their cross-sectional design did not allow to evaluate cause-effect relationship between these two conditions[15][16]. To our knowledge, our study is the first with a prospective design, which gave us the opportunity to detect a causal association.

Our data confirm the central role of sedentary lifestyle in determining the development of MetS[17] and it's in agreement with data showing an association between low physical exercise and MetS[18], [19] and supports the recommendation of exercise training programmes in people with epilepsy.

Independently of sedentary lifestyle, anti-epileptic drugs may "per se" induce metabolic disturbances prompting the development of the syndrome[20], [21].This highlight the importance of regular monitoring of AEDs and metabolic check-ups in people with epilepsy who are under anti-epileptic medication.

In this study we found many incidents of components of MetS (hyperglycemia, dyslipidemia, high abdominal obesity). These findings are in agreement with some reports which confirmed that phenytoin and valproic acid can trigger hyperglycemia in non diabetic patients[22][23] and that in general almost all AEDs induce increased the body weight[24] and disturb the lipid profile[25].

Although this study is quite informative, it has nerveless some limitations to be pointed out. There were no data on anthropometric and biochemical measurements before the patients were initiated on AEDs treatment. Additionally, although efforts were made to convince study participants to fast overnight before sample collection, we could not ascertain that all of them adhered to this recommendation.

Finally, due to budget and time constraints we followed up our cohort of participants for a period of one year only. This duration was not sufficient for the development of MetS in all participants who were at risk of developing MetS. This impacted seriously our findings to such an extent that we cannot ascertain with utmost exactitude the causal association between epilepsy and metabolic syndrome.

Nevertheless, this study lays a good foundation for further researches on other issues like interactions of AEDs and other medications in development of MetS in people with epilepsy and markers of genetic susceptibility of MetS in patients with 


\section{International Journal of Current Science Research and Review}

ISSN: 2581-8341

Volume 04 Issue 05 May 2021

DOI: 10.47191/ijcsrr/V4-i5-03, Impact Factor: 5.825

IJCSRR@ 2021

Www.ijesrr.org

epilepsy etc.

\section{Recommendations}

As it has been found, people with epilepsy are more prone to develop MetS. Therefore, it is recommended that healthcare practitioners do regular clinical and laboratory checkups on patients with epilepsy who are receiving AEDs medications in order to early diagnose MetS thereby halting its complications. We recommend that preventive measures should be taken to reduce or eliminate the development of MetS among patients with epilepsy by instilling in them the importance of regular exercises and diet modification. Also, any health problem faced should be immediately reported so than appropriate management approaches can be urgently considered.

Furthermore, given the fact the follow up duration for our prospective cohort was not adequate, larger studies with longer follow up period of at least 5 year or more are recommended in order to obtain more conclusive findings.

\section{Contributors}

All authors played a role in the study design. FXN and JBG conducted the study and collected data. FXN, BN and SPR analyzed and interpreted the data. FXN, BN and SPR wrote the manuscript and approved its final version prior to submission for publication

\section{Acknowledgment}

The authors would like to thank interviewers and nurses who participated in recruiting of study participants and data collection. The principal investigator acknowledges a financial support he got from the German Academic Exchange Service (Deutscher Akademischer Austausch Dienst, DAAD).

\section{REFERENCES}

1. K. Shimamoto, '[Metabolic syndrome and cardiovascular diseases].', Nihon Naika Gakkai zasshi. The Journal of the Japanese Society of Internal Medicine, vol. 97, no. 3. pp. 591-597, 10-Mar-2008.

2. M. Šimunovit, J. Bodit, L. Milit, I. Unit, and V. Škrabit, 'The Prevalence of Metabolic Syndrome and Cardiovascular Risk Factors in Obese Children and Adolescents in Dalmatia: A Hospital Based Study', hindawi.com, 2009.

3. E. Bonora, 'The metabolic syndrome and cardiovascular disease', Annals of Medicine, vol. 38, no. 1. Taylor and Francis A.S., pp. 64-80, 2006.

4. E. B.-A. of medicine and undefined 2006, 'The metabolic syndrome and cardiovascular disease', Taylor Fr.

5. P. H.-T. in E. \& Metabolism and undefined 2009, 'eNOS, metabolic syndrome and cardiovascular disease', Elsevier.

6. S. A. Ritchie and J. M. C. Connell, 'The link between abdominal obesity, metabolic syndrome and cardiovascular disease', Elsevier.

7. S. M. Grundy, 'Obesity, Metabolic Syndrome, and Cardiovascular Disease', academic.oup.com, 2004.

8. A. Gil-Núñez, 'The Metabolic Syndrome and Cerebrovascular Disease: Suspicion and Evidence', Cerebrovasc. Dis., vol. 24, no. 1, pp. 64-75, Nov. 2007.

9. B. Solymoss, M. Bourassa, ... J. L.-C. artery, and undefined 2003, 'Incidence and clinical characteristics of the metabolic syndrome in patients with coronary artery disease', journals.lww.com.

10. G. D. Kolovou, K. K. Anagnostopoulou, K. D. Salpea, and D. P. Mikhailidis, 'The prevalence of metabolic syndrome in various populations', American Journal of the Medical Sciences, vol. 333, no. 6. Elsevier, pp. 362-371, 01-Jun-2007.

11. S. G. Schorr, C. J. Slooff, R. Bruggeman, and K. Taxis, 'The incidence of metabolic syndrome and its reversal in a cohort of schizophrenic patients followed for one year', J. Psychiatr. Res., vol. 43, no. 13, pp. 1106-1111, 2009.

12. A. Gaitatzis, K. Carroll, A. Majeed, and J. W. Sander, 'The epidemiology of the comorbidity of epilepsy in the general population', Epilepsia, vol. 45, no. 12, pp. 1613-1622, Dec. 2004.

13. K. N.-N. treatment of epilepsy. S. Intech and undefined 2011, 'Adverse metabolic effect of antiepileptic drug treatment', books.google.com.

14. E. Goodman, S. R. Daniels, J. A. Morrison, B. Huang, and L. M. Dolan, 'Contrasting prevalence of and demographic disparities in the World Health Organization and National Cholesterol Education Program Adult Treatment Panel III 


\section{International Journal of Current Science Research and Review}

ISSN: 2581-8341

Volume 04 Issue 05 May 2021

DOI: 10.47191/ijesrr/V4-i5-03, Impact Factor: 5.825

IJCSRR@ 2021

WWw.ijcsrr.org

definitions of metabolic syndrome among adolescents', J. Pediatr., vol. 145, no. 4, pp. 445-451, Oct. 2004.

15. S. Nair, S. Harikrishnan, P. Sarma, S. T.- Seizure, and undefined 2016, 'Metabolic syndrome in young adults with epilepsy', Elsevier.

16. L. H. Ronquillo and J. Francisco Tellez-Zenteno, 'Obesity and its association with generalised epilepsy, idiopathic syndrome, and family history of epilepsy’, Wiley Online Libr., vol. 16, no. 3, pp. 343-353, Sep. 2014.

17. S. T, P. R, and L. J, 'SenseWear-determined physical activity and sedentary behavior and metabolic syndrome.', Med. Sci. Sports Exerc., vol. 45, no. 3, pp. 481-489, Mar. 2013.

18. A. Bankoski et al., 'Sedentary activity associated with metabolic syndrome independent of physical activity', Diabetes Care, vol. 34, no. 2, pp. 497-503, Feb. 2011.

19. C. L. Edwardson et al., 'Association of Sedentary Behaviour with Metabolic Syndrome: A Meta-Analysis', PLoS One, vol. 7, no. 4, p. e34916, Apr. 2012.

20. A. Verrotti, R. Manco, S. Agostinelli, ... G. C.-, and undefined 2010, 'The metabolic syndrome in overweight epileptic patients treated with valproic acid', Wiley Online Libr.

21. A. Dhir, S. Sharma, P. Jain, ... B. B.-J. of pediatric, and undefined 2015, 'Parameters of metabolic syndrome in Indian children with epilepsy on valproate or phenytoin monotherapy', ncbi.nlm.nih.gov.

22. B. L. Carter, R. E. Small, M. D. Mandel, and M. T. Starkman, 'Phenytoin-induced hyperglycemia', Am. J. Heal. Pharm., vol. 38, no. 10, pp. 1508-1512, Oct. 1981.

23. R. M. Nanau and M. G. Neuman, 'Adverse drug reactions induced by valproic acid', Clinical Biochemistry, vol. 46, no. 15. Elsevier, pp. 1323-1338, 01-Oct-2013.

24. S. A. Hamed, 'Antiepileptic drugs influences on body weight in people with epilepsy', Expert Review of Clinical Pharmacology, vol. 8, no. 1. Expert Reviews Ltd., pp. 103-114, 01-Jan-2014.

25. M. Vyas, B. Davidson, L. Escalaya, J. C.-E. research, and undefined 2015, 'Antiepileptic drug use for treatment of epilepsy and dyslipidemia: systematic review', Elsevier.

Cite this Article: François Xavier Ndayambaje, Jean Bosco Gahutu, Simon Peter Rugera, Bernard Natukunda (2021). Incidence of the Metabolic Syndrome among Patients with Epilepsy Attending a Neuropsychiatric Hospital in Kigali, Rwanda. International Journal of Current Science Research and Review, 4(5), 339-345 